\title{
Risk of Cerebral Angiography in Patients with Symptomatic Intracranial Atherosclerotic Stenosis
}

\author{
Harry J. Cloft ${ }^{a}$ Michael J. Lynn ${ }^{b}$ Edward Feldmann ${ }^{c}$ Marc Chimowitz ${ }^{d}$ \\ for the WASID Study Group \\ ${ }^{a}$ Departments of Radiology and Neurosurgery, Mayo Clinic, Rochester, Minn., ${ }^{b}$ Department of Neurology, \\ Emory University, Atlanta, Ga., 'Department of Neurology, Brown University, Providence, R.I., and \\ ${ }^{\mathrm{d}}$ Department of Neurology, Medical University of South Carolina, Charleston, S.C., USA
}

\section{Key Words}

Cerebral angiography $\cdot$ Intracranial atherosclerotic stenosis, symptomatic

\begin{abstract}
Background: A well-defined rate of adverse events following cerebral angiography in patients with symptomatic intracranial atherosclerosis would be useful to physicians making decisions regarding imaging and treatment of these patients. We report the adverse events associated with angiography in patients who underwent single-vessel cerebral angiography as part of the study protocol in the WarfarinAspirin for Symptomatic Intracranial Arterial Stenosis trial. Methods: Single-vessel cerebral angiography was performed to specifically define the degree of stenosis in 196 patients suspected of having intracranial atherosclerotic stenosis on noninvasive tests. Adverse events that occurred within $24 \mathrm{~h}$ of cerebral angiography were reported by the sites performing the angiography. Results: Overall, neurological adverse events occurred in 4 patients $(2.0 \%$; $95 \% \mathrm{Cl}$ : 0.6-5.1\%), and nonneurological adverse events occurred in 12 patients (6.1\%; $95 \% \mathrm{Cl}: 3.2-10.5 \%)$. All of the neurological adverse events were transient. Conclusions: The risk of permanent neurological adverse events associated with single-
\end{abstract}

vessel cerebral angiography in patients with symptomatic intracranial atherosclerosis is relatively low. The quantification of the risk of cerebral angiography in patients with intracranial atherosclerosis provides useful information to consider when evaluating noninvasive imaging techniques for their relative value.

Copyright $\odot 2011$ S. Karger AG, Basel

\section{Introduction}

The risks of cerebral angiography have been assessed in a number of prospective studies [1-6]. Some of these studies examined adverse event rates in all patients undergoing cerebral angiography [1-4], while others have examined the risk only in patients undergoing angiography for transient ischemic attack (TIA) or ischemic stroke $[5,6]$. The risk in patients with TIA and ischemic stroke has tended to be higher than in patients with other indications for cerebral angiography. In a meta-analysis of the risk of cerebral angiography in patients with TIA or stroke [7], the risk of transient neurological deficit was $3.0 \%$ and the risk of permanent neurological deficit was $0.7 \%$.

\section{KARGER}

(C) 2011 S. Karger AG, Basel

Fax +41613061234 E-Mail karger@karger.ch www.karger.com www.karger.com/ced
Harry J. Cloft, MD, PhD

Department of Radiology, Mayo Clinic

200 First Street SW

Rochester, MN 55901 (USA)

Tel. +1 507266 3350, E-Mail cloft.harry@ mayo.edu 
Patients with symptomatic intracranial atherosclerotic stenosis are a specific subset of patients with TIA or stroke. Noninvasive identification of intracranial stenosis in these patients by transcranial Doppler (TCD) and magnetic resonance angiography (MRA) sometimes requires catheter angiography for confirmation and measurement of the degree of stenosis $[8,9]$. Thus, catheter angiography remains a clinically important technique for patients with intracranial stenosis. The risk of cerebral angiography in this specific subset of patients has not been previously studied. We report the risk of single-vessel cerebral angiography in patients with suspected symptomatic intracranial atherosclerotic stenosis who underwent angiography as part of the study protocol in the Warfarin-Aspirin for Symptomatic Intracranial Arterial Stenosis (WASID) trial.

\section{Methods}

\section{Study Design and Patient Eligibility}

Details of the WASID study design and results of the comparison of warfarin and aspirin have been published previously [10]. Briefly, the WASID trial was an investigator-initiated, randomized, double-blind, multi-center clinical trial conducted at 59 sites in North America, which was funded by the National Institute of Neurological Disorders and Stroke. The study protocol was approved by the institutional review boards at each site, and all patients gave written informed consent to participate. Patients were enrolled between February 1999 and July 2003.

Inclusion criteria included: TIA or nondisabling stroke within 90 days of randomization that was attributable to angiographically verified $50-99 \%$ stenosis of a major intracranial artery (internal carotid, middle cerebral, vertebral or basilar artery); modified Rankin Scale score of $\leq 3$, and age of $\geq 40$ years. Exclusion criteria included: extracranial internal carotid stenosis (50-99\%) in tandem with an intracranial carotid or middle cerebral artery stenosis; nonatherosclerotic stenosis of an intracranial artery; a cardiac source of embolism (e.g. atrial fibrillation); contraindication to aspirin or warfarin, and comorbid condition limiting survival to less than 5 years. Patients were randomly assigned treatment with warfarin (target international normalized ratio between 2.0 and 3.0) or aspirin (1,300 mg daily). The study subjects and investigators were blinded to treatment allocation.

\section{Angiographic Evaluation}

Patients could only be enrolled in the WASID trial if a catheter angiogram demonstrated $50-99 \%$ stenosis of a major intracranial artery as measured by a site investigator. The angiogram could have been done at the local clinical center as part of routine care, or the patient may have been referred to the local clinical center with an angiogram already having been done. For other potential study patients who had a noninvasive test (MRA, TCD or CT angiography) that suggested $50-99 \%$ intracranial stenosis and in whom the local investigator would not typically request that an angiogram be done as part of routine clinical care, the
WASID study provided funds to cover the costs of a single-vessel angiogram. Approval for the study to pay the costs of the singlevessel angiogram had to be requested before the angiogram was done. To provide an unbiased set of patients for this report, both in terms of the selection of the patients and the reporting of the adverse events, we have included only those patients for whom a single-vessel angiogram was paid by the WASID study.

\section{Evaluation of Adverse Events after Cerebral Angiography}

The local clinical center completed case report forms (CRF) documenting the results of the angiogram. The CRF specifically requested whether or not any of the following adverse events occurred within $24 \mathrm{~h}$ of the angiogram: ischemic stroke, TIA, dissection, allergic reaction, groin hematoma, hypotension, congestive heart failure, chest pain, seizure and renal impairment. Space was provided on the CRF to write in any additional adverse events which may have occurred.

\section{Statistical Considerations}

We report the percentage of patients experiencing each specific type of adverse event along with a 95\% CI calculated using the exact binomial method. We also combined the adverse events into two categories: neurological adverse events (ischemic stroke, TIA and other adverse events judged to be neurological, e.g. headache, visual symptoms) and nonneurological adverse events (all other adverse events). We report the percentage of patients who experienced each type. Since a patient could have experienced more than one adverse event, we report the percentage of patients experiencing at least one adverse event as well as the frequency distribution of the number of adverse events that occurred per patient.

\section{Results}

For 201 patients, the WASID study provided funds for the cost of a diagnostic single-vessel angiogram. Data on adverse events following the angiogram were not available for 5 patients. Among the remaining 196 patients, 16 (8.2\%) experienced at least one adverse event within $24 \mathrm{~h}$ after the angiogram (95\% CI: 4.7-12.9\%).

Among the adverse events for which responses were specifically requested on the CRF, the following did not occur: ischemic stroke, hypotension, congestive heart failure, chest pain, seizure and renal impairment. The numbers and percentages of patients experiencing the other specifically requested adverse events were as follows: TIA - 1 patient (0.5\%; 95\% CI: 0.01-2.8\%); dissection -1 patient $(0.5 \%$; $95 \%$ CI: $0.01-2.8 \%)$; allergic reaction - 1 patient $(0.5 \%$; $95 \%$ CI: $0.01-2.8 \%)$, and groin hematoma -8 patients $(4.1 \%$; $95 \%$ CI: $1.8-7.9 \%)$.

For 6 patients (3.1\%; 95\% CI: 1.1-6.5\%), other adverse events were noted. These included 3 neurological events (visual-migrainous phenomenon that resolved in a few hours; headache and nausea; drowsiness requiring hospi- 
tal admission following angiogram) and 3 nonneurological events (right common femoral artery dye-injected subintimally; syncope on arising $10 \mathrm{~h}$ after angiogram; asymptomatic bradycardia).

Overall, neurological adverse events occurred in $4 \mathrm{pa}-$ tients (2.0\%; 95\% CI: 0.6-5.1\%) and nonneurological adverse events occurred in 12 patients (6.1\%; 95\% CI: $3.2-$ $10.5 \%)$. All of the neurological events were transient. Among the 16 patients with angiographic adverse events, 1 patient had more than one adverse event: groin hematoma and syncope on arising $10 \mathrm{~h}$ after the procedure with no residual deficit.

\section{Discussion}

We found a very low risk of neurological events within $24 \mathrm{~h}$ after single-vessel cerebral angiography was performed for suspected symptomatic intracranial atherosclerotic stenosis in this multicenter trial ( 59 sites). In particular, there were no ischemic strokes, and only 1 TIA in 196 patients.

Multiple factors have been correlated with the risk of cerebral angiography. The risk of cerebral angiography has been correlated with the age of the patient $[1,3]$, the total volume of contrast used [1], the length of the procedure $[1,2]$, the use of more than one catheter [3] and systolic hypertension [2]. One possible reason for the very low rates of neurological adverse events in this study was that angiography was limited to a single vessel.

People who undergo cerebral angiography tend to have cerebrovascular disease, which can lead to cerebrovascular events with or without cerebral angiography. Many neurological events attributed to cerebral angiography may be due to the underlying cerebrovascular disease that led to angiography rather than the performance of cerebral angiography itself. Between 24 and $72 \mathrm{~h}$ after the performance of cerebral angiography, Dion et al. [2] observed rates of $1.5 \%$ for transient ischemic events and $0.3 \%$ for development of persistent deficits, rates that were not significantly different from the rates observed in the first $24 \mathrm{~h}$ after angiography. These events at 24$72 \mathrm{~h}$ following angiography are possibly due to the natural history of a patient's cerebrovascular disease (especially emboli secondary to atherosclerosis) rather than the performance of angiography. Thus, the neurological adverse event rates in patients with cerebrovascular disease would not be 0 if no angiography, or if 'perfectly safe' angiography, could be performed [11].
The overall risk of nonneurological adverse events with angiography in our study was low, which is consistent with other studies $[1-3,7]$. In the series by Dion et al. [2], serious nonneurological conditions consisted of 5 large inguinal hematomas requiring fluid replacement or surgery. In the series by Earnest et al. [3], the serious nonneurological adverse events consisted of 2 large inguinal hematomas requiring medical or surgical therapy, 3 incidences of peripheral thromboembolic events, 5 cases of transient hypotension requiring medical therapy, 1 case of transient hypertension requiring medical therapy, and 1 case of infection. In the series by Heiserman et al. [1], there were 3 cases of transient hypotension requiring therapy. Minor hematomas occurred in $6.9 \%$ of the patients in the series reported by Dion et al. [2], and in $8.1 \%$ of the patients in the series reported by Heiserman et al. [1], but they were not reported in the series by Earnest et al. [3]. Dion et al. [2] noted that nonneurological adverse events were significantly related to age of $>50$ years, hypertension, TIA as an indication, and the presence of a carotid bruit. Since patients with symptomatic intracranial atherosclerotic stenosis share many of these risk factors, their risk of nonneurological adverse events after cerebral angiography would be expected to be higher than in the general population. In our study, it was not possible to further evaluate the data for important confounding risk factors such as patient age, length of procedure, and degree of atherosclerosis.

Advances have been made in CT angiography [12], MRA [13-15] and TCD [8] that have led some investigators to suggest that these techniques may soon replace conventional angiography in patients with symptomatic intracranial atherosclerotic stenosis. These techniques are noninvasive and therefore carry a lower adverse event rate than conventional catheter angiography, but diagnostic accuracy is sometimes a problem. For example, in the SONIA (Stroke Outcomes and Neuroimaging of Intracranial Atherosclerosis) trial, MRA and TCD identification of $50-99 \%$ intracranial stenosis carried a falsepositive rate of up to $45 \%$ [8].

For accurately identifying the presence and severity of disease and monitoring the effect of stenting, catheter angiography is sometimes required, and it will continue to be required if the SAMMPRIS (Stenting vs. Aggressive Medical Management for the Prevention of Recurrent Stroke) trial shows that stenting is superior to optimal medical therapy in patients with $>70 \%$ stenosis. Since the risk of cerebral angiography in patients with symptomatic intracranial atherosclerotic stenosis is quite low, the diagnostic accuracy of noninvasive imaging techniques

Cloft/Lynn/Feldmann/Chimowitz/ for the WASID Study Group 
will need to be very comparable to conventional angiography if they are to be used as a substitute for conventional angiography. The risk of cerebral angiography in patients with symptomatic intracranial atherosclerotic stenosis defined in this study will be useful to know in deciding the appropriateness of cerebral angiography versus other noninvasive techniques in the evaluation of this high-risk disease.

\section{Acknowledgments}

This study was funded by a research grant (1R01 NS36643; principal investigator: M.I. Chimowitz) from the US Public Health Service, National Institute of Neurological Disorders and Stroke.

\section{References}

1 Heiserman JE, Dean BL, Hodak JA, Flom RA, Bird CR, Drayer BP, Fram EK: Neurologic complications of cerebral angiography. AJNR Am J Neuroradiol 1994;15:1408-1411.

-2 Dion JE, Gates PC, Fox AJ, Barnett HJ, Blom RJ: Clinical events following neuroangiography: a prospective study. Stroke 1987;18:9971004.

- 3 Earnest F, Forbes G, Sandok BA, Piepgras DG, Faust RJ, Ilstrup DM, Arndt LJ: Complications of cerebral angiography: prospective assessment of risk. AJR Am J Roentgenol 1983;142:247-253.

-4 Waugh JR, Sacharias N: Arteriographic complication in the DSA era. Radiology 1992;182:243-246.

5 Hankey JG, Warlow CP, Molyneux AJ: Complications of cerebral angiography for patients with mild carotid territory ischaemia being considered for carotid endarterectomy. J Neurol Neurosurg Psychiatry 1990;53: 542-548.

6 Hankey GJ, Warlow CP, Sellar RJ: Cerebral angiographic risk in mild cerebrovascular disease. Stroke 1990;21:209-222.
7 Cloft HJ, Joseph GJ, Dion JE: Risk of cerebral angiography in patients with subarachnoid hemorrhage, cerebral aneurysm, and arteriovenous malformation: a meta-analysis. Stroke 1999;30:317-320.

8 Feldmann E, Wilterdink JL, Kosinski A, Lynn M, Chimowitz MI, Sarafin J, Smith HH, Nichols F, Rogg J, Cloft HJ, Wechsler L, Saver J, Levine SR, Tegeler C, Adams R, Sloan $\mathrm{M}$, Stroke Outcomes and Neuroimaging of Intracranial Atherosclerosis (SONIA) Trial Investigators: Stroke outcomes and neuroimaging of intracranial atherosclerosis (SONIA) trial. Neurology 2007;68:2099-2106.

-9 Saba L, Sanfilippo R, Montisci, Mallarini G: Assessment of intracranial arterial stenosis with multidetector CT angiography: a postprocessing techniques comparison. AJNR Am J Neuroradiol 2010;31:874-879.

10 Chimowitz MI, Lynn MJ, Howlett-Smitt H, Stern BJ, Hertzberg VS, Frankel MR, Levine SR, Chaturvedi S, Kasner SE, Benesch CG, Sila CA, Jovin TG, Romano JG, WarfarinAspirin Symptomatic Intracranial Disease Trial Investigators: Comparison of warfarin and aspirin for symptomatic intracranial arterial stenosis. N Engl J Med 2005;352:13051316.

-11 Baum S, Stein GN, Kuroda KK: Complications of 'no arteriography'. Radiology 1966; $86: 835-838$.
12 Bash S, Villablanca JP, Jahan R, Duckwiler G, Tillis M, Kidwell C, Saver J, Sayre J: Intracranial vascular stenosis and occlusive disease: evaluation with CT angiography, MR angiography, and conventional angiography. AJNR Am J Neuroradiol 2004;25:1012-1021. 13 Hirai T, Korogi Y, Ono K, Nagano M, Maruoka K, Uemura S, Takehashi M: Prospective evaluation of suspected stenoocclusive disease of the intracranial artery: combined MR angiography and CT angiography compared with digital subtraction angiography. AJNR Am J Neuroradiol 2002;23:93101.

14 Korogi Y, Takahashi M, Nakagawa T, Mabuchi N, Watabe T, Shiokawa Y, Shiga $\mathrm{H}$, O'Uchi T, Miki H, Horikawa Y, Fujiwara S, Furuse M: Intracranial vascular stenosis and occlusion: MR angiographic findings. AJNR Am J Neuroradiol 1997;18:135-143.

15 Alvarez-Linera J, Benito-Leon J, Escribano J, Campollo J, Gesto R: Prospective evaluation of carotid artery stenosis: elliptic centric contrast-enhanced MR angiography and spiral CT angiography compared with digital subtraction angiography. AJNR Am J Neuroradiol 2003;24:1012-1019. 